\title{
Spectral Ensemble Clustering via Weighted K- Means: Theoretical and Practical Evidence
}

\author{
R.Dinesh Kumar, M.Srinivasalu, T.Upender, G.Ramya, K.Pratap
}

\begin{abstract}
As a promising avenue for investigation of heterogeneous information, bunching agreement has been interesting in expanding consideration in the ongoing decade. Among the major different arrangements, co-affiliation lattice based structural engineering milestone, which is reclassified in accordance grouping as an issue in the segment graph. All things considered, the complexity of existence generally high block of native broad application. We propose in this manner Ensemble Spectral Clustering (SEC) to use the benefits of co-affiliation grid coordinate data have not run all the more adept. We unveil a hypothetical comparability between the SEC and the weighted $\mathrm{K}$ implies grouping, which drastically reduces the multifaceted nature of algorithmic. We also determine idle capacity SEC deal, the best information is the first to link technical co-affiliation grid-based strategy with target capacity worldwide express. Furthermore, we demonstrated in principle that the SEC holds sincerity, generalization and assembling properties. We at long last stretch out the SEC to deal with difficulties arising from inadequate important segment, given the division column conspired to great information proposed grouping. Checks on the correct indexes of different information in both clothing and multi-view shows the prevalence SEC bunching situation on some of the best in class strategies. In particular, the SEC is by all accounts a promising contender for a very large grouping information.
\end{abstract}

Keywords:Linear programming,Clustering algorithms, Partitioning algorithms, Robustness, Convergence, Complexity theory, Big data

\section{INTRODUCTION}

Accord grouping, in any other case known as troupe bunching, rises as a promising path for multi-supply, heterogeneous records grouping, and as of overdue attracts in growing scholarly consideration. It intends to locate a solitary section that typically consents with special present crucial ones [1]. It is of perceived blessings in producing effective parcels, finding novel structures, looking after uproarious highlights, and incorporating preparations from specific assets [2].Commonly, settlement grouping may be usually separated into classifications, i.E., those withor with out explicit international goal capacities. Techniques that do not set goal functionsmake usage of a few heuristicsor Meta

Revised Manuscript Received on 14 September, 2019.

R.Dinesh Kumar, Professor, Department of CSE, Siddhartha Institute of Technology \& Sciences, Narapally, Ghatkesar, Hyderabad, Telangana, India.

M.Srinivasalu, Assocate. Prof, Department of CSE, Kings Engineering College,Irungattukotta, Tamilnadu, India

T.Upender, Assist. Prof, Department of CSE, Siddhartha Institute of Technology \& Sciences, Narapally, Ghatkesar, Hyderabad, Telangana, India.

G.Ramya, Assist. Prof, Department of CSE, Siddhartha Institute of India.

K.Pratap, Assist. Prof, Department of CSE, Siddhartha Institute of Technology \& Sciences, Narapally, Ghatkesar, Hyderabad, Telangana, India. Technology \& Sciences, Narapally, Ghatkesar, Hyderabad, Telangana,

heuristics to find out surmised preparations. Agent techniques comprise consociation lattice based totally completely techniques, diagram based completely calculations, relabeling and casting a ballot strategies and regionally flexible bunch based totally totally strategies [10]. Strategies with unequivocal locations make use of global goal capacities to quantify the contrast among critical allotments and the accord one. Agent arrangements with severa target capacities include okay-implies like calculation, NMF], EM calculation, reenacted toughening and blend regularization. Of the previously mentioned strategies, the co-affiliation network based techniques form a milestone. With the ascending of records sharing websites, for example, facebook and Flickr, there is a sensational development in the amount of records. As an instance, facebook evaluations around 6 billion new photo every month and seventy two hours of video are transferred to YouTube constantly. Considered one of large statistics mining errands is to solo order the sizeable scale facts [Biwa's and Jacobs, 2012; Lee and Grumman, 2009; Deck and Frey, 2007; Cai et al., 2011], that is useful for some statistics healing and association programs.

\section{PROPOSED GADGET \& METHODOLOGY}

The least tough route is to link multi-see consists of straightforwardly and direct fashionable bunching by using manner of streamlining positive misfortune capacities utilising okay-implies and using Non-awful network factorization. Another amassing of calculations imply to discover a widespread low-measurement inert subspace, which shares the maximum achievable consistency between numerous views.

Any bunching calculations may want to then be able to be finished to accumulate the remaining parcel. As an example proposed Collective NMF to locate a mutual coefficient framework with severa premise networks crosswise over views, and comparably utilized standard to gather a common marker matrix.We anticipated the Spectral Ensemble Clustering (SEC) calculation. With the aid of recognizing the proportional connection among SEC and weighted kimplies, we diminished the fact complexities of SEC appreciably. The inborn accord target functionality of SEC changed into likewise uncovered, which connects the coaffiliation network based totally strategies with the strategies with explicit international target capacities. We at that thing researched the heartiness, generalizability and meeting houses of SEC to characteristic its predominance in principle, and stretched out it to address fragmented essential allotments 


\section{SPECTRAL ENSEMBLE CLUSTERING VIA WEIGHTED K-MEANS: THEORETICAL AND PRACTICAL EVIDENCE}

\section{Favorable instances}

- We at closing stretch out SEC to deal with the problem rising from insufficient important allotments, in light of which a line branch plot for sizable facts grouping is proposed. - This explains some non-unimportant downsides, especially while confronting informational indexes of numerous attributes.

Writing

Multi-View k-method Clustering on massive data

Xiao Cai, FeipingNie, Heng Huang*college of Texas at ArlingtonArlington, Texas, 76092

In beyond decade, an ever developing quantity of facts are accumulated from numerous resources or spoke to by means of numerous views, where diverse perspectives painting unmistakable factors of view of the statistics. Albeit every view can be independently applied for discovering designs by means of grouping, the bunching execution could be increasingly more specific with the aid of manner of investigating the rich statistics amongst numerous views. Some multi-see grouping strategies had been proposed to solo coordinate numerous views on data. Though, they're chart based methodologies, for example in mild of phantom grouping, with the give up purpose that they can not address the massive scale records. Commands to join these heterogeneous highlights for solo massive scale facts bunching has grow to be a difficult trouble. In this paper, we recommend each other hearty big scale multi-see grouping method to include heterogeneous portrayals of large scale information. We examine the proposed new techniques by using six benchmark informational indexes and contrasted the exhibition and some normally utilized bunching procedures simply because the gauge multi-see grouping techniques. In every exploratory final results, our proposed strategies reliably accomplish advanced'sclubs.

Social studying via Collective Matrix Factorization

Ajit P. Singh gadget getting to know branch Carnegie Mellon college Pittsburgh, PA 15213

Geoffrey J. Gordon machine learning department Carnegie Mellon college Pittsburgh, PA 15213

Social studying is involved approximately looking forward to hard to recognize estimations of a connection, given a database of things and watched members of the family among elements. A case of social gaining knowledge of is movement image score expectation, in which factors have to include customers, movies, types, and on-show characters. Members of the family encode customers' appraisals of motion pics, motion images' training, and entertainers' jobs in movement snap shots. A general forecast technique given one pairwise connection, as an example a \#customers $\times$ \#films critiques framework, is lowposition grid factorization. In regions with numerous own family participants, spoke to as exceptional grids, we can also decorate prescient exactness via manner of abusing statistics from one connection even as anticipating every other. To this stop, we endorse an combination lattice factorization model: we at the equal time component a few grids, sharing parameters amongst elements when a substance takes an hobby in numerous family members. Each connection could have an change actually really worth kind and blunder movement; alongside the ones strains, we allow nonlinear connections the various parameters and yields, utilizing Bergman divergences to quantify mistake. We stretch out modern-day rotating projection calculations to our version, and infer an effective Newton replace for the projection. Furthermore, we advocate stochastic improvement strategies to manipulate large, inadequate lattices. Our model sums up a few gift lattice factorization techniques, and thusly yields new massive scale enhancement calculations for those problems. Our version can deal with any pairwise social blueprint and a extensive collection of mistake models. We showcase its effectiveness, honestly because the gain of sharing parameters among members of the family.

\section{Photo department}

In advanced picture getting equipped, department is massive for picture portrayal and order. Bunches may be framed for pix boom on pixel pressure, shading, floor, place, or a combination of those. "Unearthly bunching includes the Eigen deterioration of a pair insightful similitude network, it really is unmanageable for appropriately massive pix. Scaling down the photograph, however, will cause misplaced higher subtleties and may prompt incorrect department consequences" (Tung, Wong, and Clause, 2010). So Tung et al. (2010) [7] proposed a method for ghastly grouping to large photos utilizing a aggregate of square insightful preparing and stochastic accumulating accord. The opportunity of this technique is to play out an overbranch of the photograph at the pixel level utilizing unearthly grouping, and afterward consolidate the sections utilizing a combination of stochastic outfit settlement and a 2 nd round of ghastly bunching at the fragment degree. Also, we employ stochastic troupe accord o contain every global and network image attributes in figuring out the pixel characterizations. This improvement moreover evacuates square astute coping with historic rarities. (Tung et al., 2010) Tung et al. (2010)[7] likewise exhibited the trial effects on numerous common scene pix (from the Berkeley department database) of the standardized lessen, oneself tuning ghostly grouping. They presume that "the proposed strategy accomplishes department outcomes which can be almost equal to or advanced to the next strategies. Mainly, issue by using point structures are better stored within the branch, as pondered within the better compare esteems"

Past due improvements IN SPECTRAL CLUSTERING set of rules

Over the previous decade, unearthly grouping techniques have picked up occurrence as a way to carry out facts bunching one of the maximum essential errands of AI. Those strategies admire some enormous elements of interest, as an instance, the capability to bunch nonvectorial records, and regularly yield primary specific execution. Also, they may be properly-pondered and upheld hypothetically. In our writing examine we outstanding the massive advances in the phantom grouping calculation. Right here we are telling a part of the enhancements.

Improvement in time unpredictability Anna Choromanska 1.TonyJebara2, Hyungtae Kim

2. Mahesh Mohan

Published By: 


\section{Moreover, Claire Monteleoni}

Suggest and wreck down a quick ghostly bunching calculation with computational multifaceted nature without delay in the amount of facts shows that is legitimately appropriate big scale datasets. The calculation consolidates ground-breaking methods in AI: ghostly grouping calculations and Nystrom methods[8] commonly used to get brilliant top notch low role approximations of extensive frameworks. The proposed calculation applies the Nystrom wager to the chart Laplacians to carry out bunching. We deliver hypothetical research of the presentation of the calculation and show the error sure it accomplishes and we speak approximately the conditions underneath which the calculation execution is nearly same to otherworldly grouping with the primary diagram Laplacians

Life green Spectral Clustering through Column Sampling Li1 ,Xiao-Chen Lian1,James T. Kwok2 and Baoliang Lu1[9] As only a few eigenvectors are required in the technique, a stylish way to address mitigate this problem is by means of the use of the usage of low-role community approximations, among which the Nystrom method

\section{Conversation of The Parameter}

We employ one parameter $\gamma$ to manipulate the float of weight elements for severa views. From Eq. (17), we will see that after $\gamma \rightarrow \infty$, we're able to get equivalent.

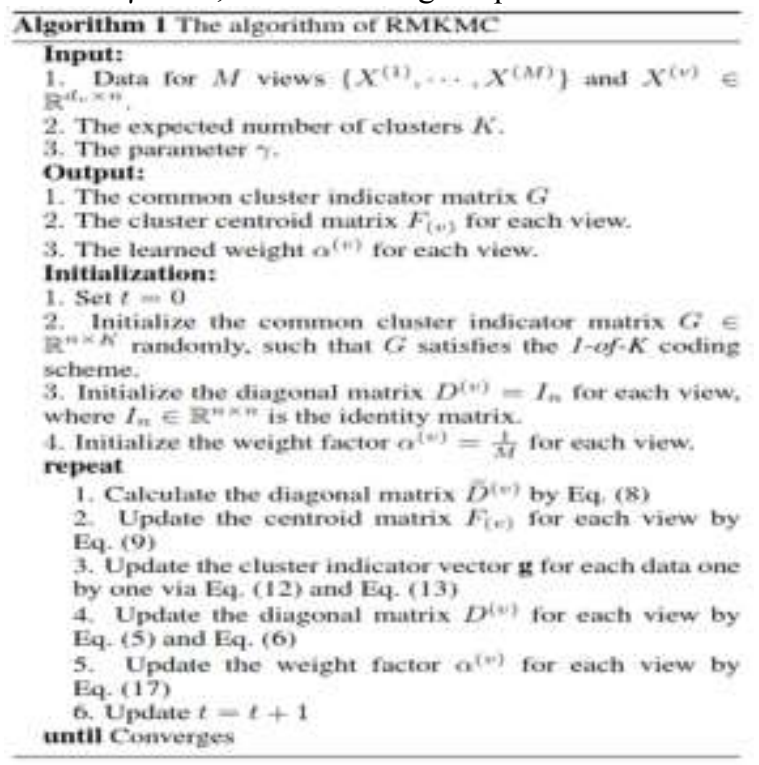

We can prove the convergence of the proposed Alg. 1 as follows: We can divide the equation. (2) into four subproblems and each of them is a matter convex to one variable. Therefore, to solve the problem of sub alternative, our pro posed algorithm will ensure that we can find the optimal solution for each sub-problems and eventually, the algorithm will converge to a local solution.

Table 1: Data set summary.

\begin{tabular}{c|c|c|c}
\hline Data sets & \# of data & \# of views & \# of cluster \\
\hline SensIT & 300 & 2 & 3 \\
\hline Caltech7 & 441 & 6 & 7 \\
\hline MSRC-v1 & 210 & 6 & 7 \\
\hline Digit & 2000 & 6 & 10 \\
\hline AwA & 30475 & 6 & 50 \\
\hline SUN & 10000 & 7 & 100 \\
\hline
\end{tabular}

Is $\mathrm{P}$, then the time intricacy is $\mathrm{O}(\mathrm{P} \mathrm{Knd})$ and the time multifaceted nature of our proposed approach is $\mathrm{O}(\mathrm{P} \mathrm{KndM}$ ), wherein $\mathrm{M}$ is the amount of views and commonly $\mathrm{P} n, \mathrm{M}$ $\mathrm{n}$ and ok $\mathrm{n}$. Likewise, in the true usage, if the information is certainly too huge to keep them in memory, we can extend our calculation as an outside reminiscence calculation that takes a shot at a lump of statistics proper away and repeat the proposed calculation on each datum piece in parallel if particular processors are handy. When the entirety of the information lumps were prepared, the group centroid community will be refreshed. Therefore, our proposed method can be utilized to cope with the exceedingly huge scale bunching problem.Due to the fact the chart primarily based totally multi-see grouping strategies cannot be applied to the significant scale image grouping, we did not assessment the presentation of our strategy and them inside the examinations.

\section{RELATED WORK}

While all is stated in completed, our approach learns a full of existence portrayal for the consociation grid thru the low-position critical, therefore it is firmly identified with collecting grouping and espresso-feature lattice research. On this while all is stated in carried out, our technique learns a strong portrayal for the consociation grid through the lowcharacteristic requirement, therefore it's far firmly diagnosed with outfit grouping and low-function lattice research. On this phase, we will provide a concise presentation for those factors. 2.1 Ensemble Clustering extremely good endeavors had been made to fathom outfit bunching. The leaving paintings can be commonly remoted into instructions as following. The essential beauty makes use of an application ability to quantify the similitude many of the accord bunching and numerous BPs, and for the maximum component finds the final parcel via amplifying an explicit goal paintings. For instance, Torchy et al. [27] proposed a Quadratic Mutual statistics primarily based aim artwork for settlement grouping, and carried out ok-implies bunching to find out the association. They in addition stretched out their work to utilizing the EM calculation with a confined mixture of multinomial disseminations for accord bunching [28]. Along this line, $\mathrm{Wu}$ et al. [32] moved the corporation bunching into a k-implies grouping issue with KCC utility functionality and gave the important and ok conditions for KCC application capacities. What's greater, there are some extraordinary charming target capacities for the group bunching, for example, those depending on nonnegative lattice factorization [12], piece based techniques [30], and recreated toughening [18], one by one. The following class abridges the statistics of facts BPs proper right into a coaffiliation network, which exams how regularly examples seem in a comparable bunch. The co-affiliation framework truely speaks to the pairwise comparison of the vast amount of records focuses in the segment space. In this manner, a diagram parcel calculation may be directed on it to accumulate the remaining grouping outcome. Strehl and Ghosh [26] created 3 chart based completely calculations for

Published By: 
accord bunching, at the equal time as Fred and Jain [8] implemented the agglomerative modern grouping. As of past due, Liu et al. [16] proposed a ghastly amassing grouping approach, which ran otherworldly bunching at the co-association lattice and changed it as a weighted okayimplies trouble to perform immoderate effectiveness. Distinctive techniques include Relabeling and balloting [1], locally Adaptive Cluster primarily based totally techniques [6], hereditary calculation based totally strategies [38], and nevertheless a few more. Anyhow, each this sort of techniques above for the most aspect legitimately consolidate numerous BPs without thinking about approximately the clamors. Little exertion has been made to look for a hearty co-association community [36, 39], on this manner we endorse the RSEC strategy to address this check

\section{Low-Rank Matrix evaluation}

Low-function framework research, as a effective tool to get better easy facts from the examples containing errors (i.E., commotion, lacking passages, defilements and exceptions), has been generally applied in the fields of AI, facts mining, and computer vision. More often than not, it has agent frames as strong PCA [31, 4] and occasionalcharacteristic representation (LRR) [15, 14]. Given a watched facts community $X=[\mathrm{x} 1, \cdots, \mathrm{xn}] \in \mathrm{Rd} \times \mathrm{n}$, in which every segment vector $x i \in \mathrm{Rd}$ speaks to an example, robust PCA $[31,4]$ disintegrates $X$ proper right into a low-position lattice An and an inadequate one, which recuperates the right statistics and mistakes, in my opinion. It facilities around the challenge of low-position lattice recuperation and completing, and infers the data originate from one single subspace. Specific close to robust PCA, LRR [15, 14] receive the records are drawn from an affiliation of numerous low-dimensional subspaces, and tries to get better these subspaces via seeking out the most minimal function portrayal $Z$ for $X$ as: $\min Z, E \operatorname{rank}(Z)+\lambda E 0$ s.T. $X=X Z+$ $\mathrm{E}$,

\section{EVALUATION RESULT}

\section{Investigations}

We reflect onconsideration on each of our coregularization based multi-see ghastly bunching methodologies with various baselines. Specifically, we assessment and:

- unmarried View: the use of the most instructive view, i.E., one which accomplishes the high-quality ghostly grouping presentation the use of a solitary attitude on the information.

- characteristic Concatenation: Concatenating the highlights of each view, and later on going for walks favored otherworldly grouping making use of the chart Laplacian have been given from the joint view portrayal of the information.

- Kernel Addition: Combining various parts thru which includes them, and in a while taking walks standardspectral grouping at the regarding Laplacians. As advocated in before discoveries [9], even this reputedly straightforward method frequently activates near perfect outcomes even as contrasted with increasingly cutting-edge methodologies for order. It has an inclination to be noticed that thing growth decreases to encompass connection for the brilliant example of straight bit. While all is stated in carried out, piece growth is same as hyperlink of highlights in the Reproducing Kernel Hilbert space.

- Kernel Product (factor smart): Multiplying the touching on passages of additives and applying famous unearthly bunching on the resultant Laplacians. For the first rate example of Gaussian bit, element astute bit item could be same as straightforward element hyperlink if the 2 bits utilize identical width parameter $\sigma$. Be that as it may, in our checks, we make use of numerous width parameters for numerous views so the exhibitions of bit object might not be legitimately much like spotlight connection.

- CCA based totally completely function Extraction: making use of CCA for consist of mixture from numerous views at the statistics [10], and in a while taking walks ghastly bunching making use of those separated highlights. We observe each fashionable CCA and detail CCA for highlight extraction and report the bunching results for whichever offers the superb execution.

- Minimizing-confrontation Spectral Clustering: Our very last sample is the limiting distinction way to cope with unearthly bunching [11], and is possibly maximum firmly recognized with our co-regularization primarily based definitely manner to deal with ghostly grouping. This calculation is pointed out extra in Sec. 4. To apprehend the consequences of our co-regularization primarily based definitely methodologies, within the tables containing the outcomes, we use picture " $\mathrm{P}$ " to suggest the pairwise coregularization approach and image " $\mathrm{C}$ " to mean the centroid primarily based co-regularization technique. For datasets with multiple perspectives, we've got additionally expressly referenced the amount of views in parentheses.We document test consequences on engineered and 3 actual global datasets. We deliver a concise depiction of every dataset right here.

The results for all datasets are appeared in desk 1. For 2see manufactured facts (artificial information 1), both the co-regularized unearthly bunching methodologies outflank every one of the baselines through a vital aspect, with the pairwise method showing development over the centroidprimarily based technique. The closest acting methodologies are component expansion and CCA. For manufactured facts, request 2 polynomial piece based totally bit CCA gives nice execution amongst all CCA variations, while Gaussian issue primarily based absolutely bit CCA plays ineffectively. We do no longer file consequences for Gaussian piece CCA right here. All of the multi-see baselines outflank the single view case for the manufactured information.For three-see engineered records (artificial statistics 2), we will see that honest thing link does not guide lots. In reality, it lessens the exhibition while the $1 / 3$ view is protected, so we document the presentation with actually two perspectives for highlight link. Component boom with three views offers a exquisite improvement over unmarried view case. Whilst contrasted with unique baselines (with perspectives), both our coregularized otherworldly grouping methodologies with 
perspectives perform higher. For the two methodologies, growth of zero. 33 view furthermore brings approximately enhancing the presentation beyond the 2 view case.For the archive bunching results on Reuters multilingual records, English and French dialects are applied as the 2 perspectives. On this dataset as properly, both our methodologies beat every one of the baselines thru a crucial aspect. The following exceptional exhibition is completed with the aid of manner of least distinction ghastly grouping [11] approach. It ought to be noticed that CCA and element savvy piece item exhibitions are greater terrible than that of single view.For Caltech-one hundred and one statistics, we can not do highlight link for the reason that definitely factors are to be had. Shockingly, on this dataset, each one of the baselines perform extra horrible than the unmarried view case. Then over again, each of our co-regularized phantom bunching strategies with perspectives beat the single view case. As we covered more perspectives that were available for the Caltech-one zero one datasets, we discovered that the presentation of the pairwise technique reliably went up as we blanketed the 0.33 and the fourth view. Then again, the exhibition of the centroid-prepare approach marginally deteriorated with respect to collectively with the $1 /$ three view (likely because of the view being loud which induced the informed $\mathrm{U} *$ ); besides growth of the fourth view brought the presentation nearly near that of the pairwise case.

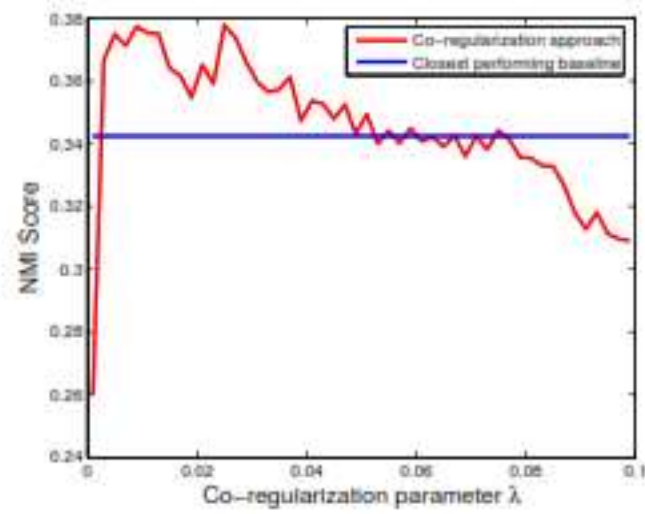

(a)

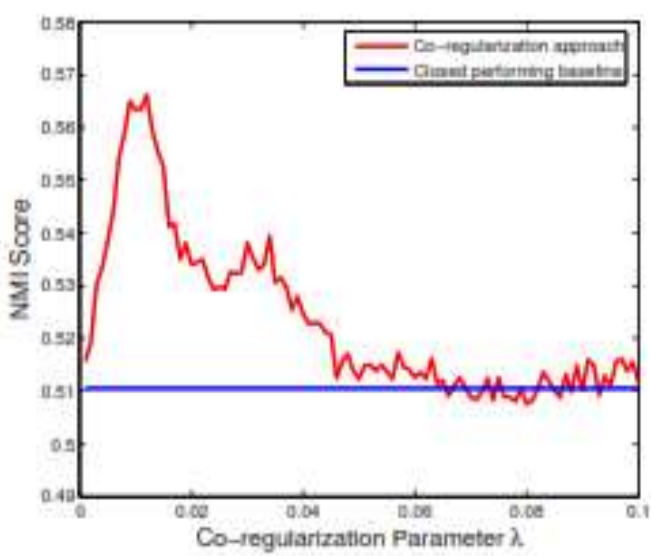

(b)

Figure1:NMIscoresofCoregularizedSpectralClusteringasafunctionof $\lambda$ for(a)Reute rsmultilingual dataand(b)Caltech-101data

\section{RMKMC METHOD \& RESULTS}

In this segment, we will examine the performance of the seasoned posed RMKMC technique to a information set of six requirements: [. Li et al 2007] SenseIT Vehicles [Duarte and $\mathrm{Hu}, 2004]$, the Caltech-101, Microsoft Research Cambridge Volume 1 (MSRC-v1) [Winn and Jojic 2005] handwritten numbers [Frank and Asuncion, 2010], Animal with attributes [Lampert et al., 2009] and GS 397 [Xiao et al., 2010]. Three preferred grouping evaluation metrics used to degree the performance of a cluster of multi-view, particularly, Clustering Accuracy (ACC), Normalized Mutual Information (NMI) and Purity.

\section{Data Set Description}

We summarize the six sets of statistics that we are able to use in our experiments in Table 1. Vehicles SenseIT records set is one of a allotted wireless sensor networks (WDSN). It uses two one of a kind sensors, namely, acoustic and seismic sensors to report special signals and carry out a type for 3 kinds of motors in wise delivery systems. We downloaded the statistics compiled from LIBSVM [Chang and Lin, 2011] and a random pattern of 100 records for every class Therefore, we've got 300 samples of information, 2 and 3 class view.

Caltech101 records set is a set of object popularity records that carries 8677 pictures, one hundred and one belong to the class. We selected a extensively used elegance 7, namely Faces, Motor, DollaBill, Garfield, Snoopy, StopSign and Windsor-Chair. Here [Dueck and Frey, 2007], we sample the facts and absolutely we've got 441 pix. In order to get a special view, we extract LBP [Ojala et al., 2002] with the scale 256, HOG [Dalal and Triggs, 2005] with the dimensions one hundred, GIST [Oliva and Torralba 2001] with the size 512 and whilst the color (CMT ) [Yu et al. 2002] with the scale forty eight, centric [Wu and Rehg 2008] with the dimensions of 1302 Dog-SIF [Lowe, 2004] with the dimensions 128 visible features of each image.

MSRC-v1 facts set is a information set that contains a scene popularity eighth grade, 240 photographs in total. Here [Lee and Grumman 2009], we pick the 7 lessons of trees, buildings, aircraft, cow, face, automobile, bicycle and each class has 30 pics. We also take the same six visible capabilities of every picture with Caltech101 dataset.

Handwritten set of numeric records that consisted of 2000 information points for zero-nine grade ten digits. (Each class has 2 hundred statistics points.) We use 6 capabilities issued to carry out multi-view clustering. In particular, this selection 6 seventy six Fourier coefficients of the form of characters (FOU), 216 correlation profiles (FAC), sixty four Karhunenlove coefficient (KAR), aver- age of 240 pixels in a $2 \times 3$ windows (PIX), forty seven Zernike moments (ZER) and6 morphology (MOR) feature.

\section{MODEL AND PARAMETER OPTIMIZATION}

For consistency, we control loads of models and parameter optimization during the test. Rated responsibility of all relationships in binary, so we used a logistic model: 

EVIDENCE

link sigmoid with matching log-loss. To evaluate the check error that we use the common absolute blunders (MAE) for a $2 \mathrm{~d}$ activity, that's the average of the 0-one loss to the binary prediction. Since the data for israted very unbalanced in choose Images aren't being judged, we weigh scale entries down through the fraction of the observed relationship where the connection become real. We use the '2 regularization whole. Unless different-wise stated regularizes all $\mathrm{G}(\mathrm{U})$ = one zero five $\mathrm{U}$ direction looking Armijo, refused date up to the length of the small step of $\eta=$ 2 -four. In the Newton step, we run to exchange the education loss drops underneath $5 \%$ of the intention. Using stochastic Newton, we run for a hard and fast quantity of iterations. (a) Ratings

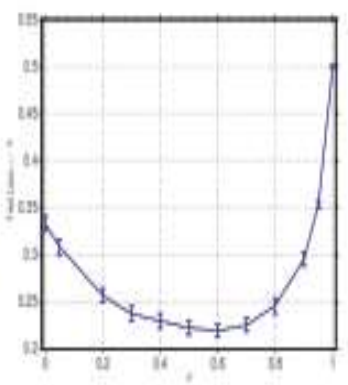

(b) Genres
Testerrors(MAE)forpredictingwhether aImagewasrated,andthegenre,on thedenserating example.

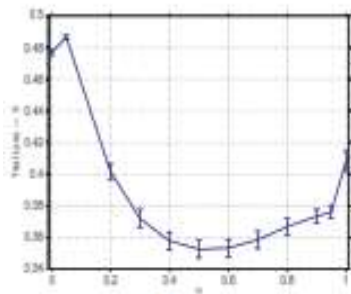

(a) Ratings

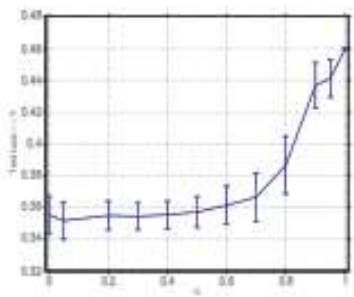

(b) Genres
Test errors (MAE) for predicting whether a Image was rated, and the genre, on sparse rating example

This calculation intertwines some assorted crucial allotments produced with the aid of normal grouping calculations right into a coordinated parcel. It has been broadly perceived that agreement bunching is strong to supply sturdy grouping effects, understand uncommon bunches, deal with commotion, exceptions, and check types, and coordinate arrangements from diverse dispersed wellsprings of data or characteristics [6] . Not in any respect just like the conventional bunching techniques which make use of the first records, the contribution of accord grouping is lots of vital partitions.Consensus bunching is a mixture trouble usually, as opposed to a standard grouping difficulty. It very well may be usually separated into two training: The predominant category plans an software capacity that estimates the likeness among fundamental segments and the last phase, and takes care of a combinatorial development problem by augmenting the application capacity [6,7]. The next magnificence makes use of a co-affiliation network to compute the activities multiple instances co-taking place in

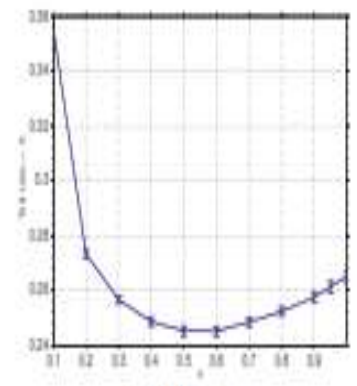

a comparable group, and in a while runs a diagram parcel method for the final agreement result. Because of the KCC [6] , we are able to exactly trade the settlement bunching with clean reduce software ability into antique fashion $\mathrm{K}$ implies grouping by supplying the accompanying parallel network. Let $\mathrm{B}=\mathrm{b}(\mathrm{x})$ be a twofold dataset gotten from the association of $r$ essential allotments $H$ as pursues: Figure 2 shows a case of the paired grid in image Segmentation by means of Saliency-Guided Constrained Clustering with Cosine Similarity. In this manner, BPs age and general are key strides for conceiving a a hit EC calculation Generally, we may produce one of a kind BPs by 3 systems [8]:

\section{Group gatherings}

The difficulty of becoming a member of numerous apportioning of loads of articles right into a solitary solidified grouping with out attending to the highlights or calculations that decided these dividing. We to begin with apprehend a few utility situations for the consequent 'facts reuse' structure that we call bunch organizations. The organization outfit issue is then formalized as a combinatorial streamlining problem as a long way as shared not unusual information. Notwithstanding a right away enlargement technique, we propose 3 a hit and efficient techniques for acquiring first rate combiners (agreement capacities). The first combiner instigates a likeness measure from the dividing and later on loners the articles. The $2 \mathrm{~d}$ combiner relies upon on hyper chart parceling. The $1 / 3$ one breakdown gatherings of agencies into meta-bunches which at that point vie for every article to determine the joined grouping. Because of the low computational expenses of our techniques, it is very possible to utilize a supra-accord work that assesses each one of the three methodologies in opposition to the target ability and picks the quality solution for a given situation. We assess the viability of bunch outfits in three subjectively awesome application conditions: (I) in which the first corporations had been framed depending on non-indistinguishable preparations of highlights, (ii) where the first bunching calculations took a shot at nonindistinguishable arrangements of articles, and (iii) wherein a normal informational index is utilized and the precept cause for consolidating numerous grouping's is to improve the nice and heartiness of the arrangement. Promising outcomes are gotten in every of the three instances for engineered simply as proper informational indexes.

Presentation The concept of incorporating special records resources and additionally discovered fashions is found in a few orders, for example, the consolidating of estimators in econometrics (Granger, 1989), proves in rule-based totally frameworks (Barnett, 1981) and multi-sensor records aggregate (Dasarathy, 1994). A simple but powerful form of multi-student framework is a set wherein every section pupil (normally a relapse oror classifier) attempts to realize a comparable undertaking. While early investigations on consolidating different ratings, for example, the works by Borda and Condorcet, pre-date the French Revolution (Ghosh, 2002a), this region perceptibly sprung up within the 
preceding decade, and now even flaunts its very own association of committed workshops (Kittler and Roli, 2002). Up to this point the primary objective of organizations has been to enhance the precision and heartiness of a given order or relapse undertaking, and of informational indexes (Sharkey, 1999). In evaluation to association or relapse settings, there have been now not many methodologies proposed for consolidating specific clusterings. 1 Notable special cases encompass:

Our case with respect to social statistics is that aggregate factorizationyields desired forecasts over utilizing a solitary grid. We take into account the is evaluated mission on two normally little informational indexes, toallow for rehashed preliminaries. Since this undertaking in-valves a 3 element version there may be a solitary mixing element, $\alpha$ in Equation 3. We benefit talent with a version for a few estimations of $\alpha$, starting from a similar beginning irregular parameters, making use of full Newton steps. The exhibition on a check set, sections inspected from the lattices as indicated by way of the test masses, is envisioned at each $\alpha$. Every initial is rehashed multiple times to give 1-trendy deviation mistake bars. Two situations areconsidered. To begin with, where the clients and movies have been inspected always at arbitrary; all training that take place in over $1 \%$ of the movement pictures are held. We simply utilize the customers' critiques on the tested movement images. Second, wherein we just sampleusers that appraised all things taken into consideration 40 movies, which fairly lessens the amount of value determinations for every customer and each movement photograph. In the number one case, the center range of evaluations in line with consumer is 60 (the mean, 127); inside the subsequent case, the center number of appraisals consistent with consumer is nine (the imply, 10). In the number one case, the center quantity of value determinations per motion photo is nine (the suggest, 21); inside the next case, the center range of critiques in line with movie is two (the imply, eight). In the primary case we have $\mathrm{n} 1=500$ clients and $\mathrm{n} 2=3000$ motion pictures and within the 2 nd case we've $n 1=750$ clients and $\mathrm{n} 2=$ one thousand motion pics. We make use of $\mathrm{a} k=20$ putting in size for the 2 frameworks.

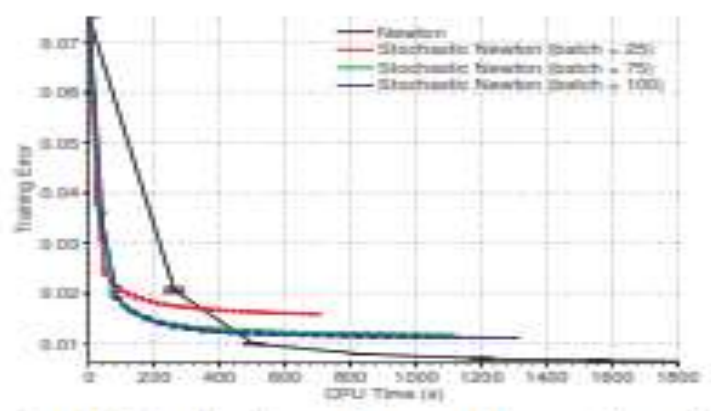

(a) Training Loss (Log-loss) remarkable enhancements were gotten for a huge assortment

\section{Relations Improve Predictions}

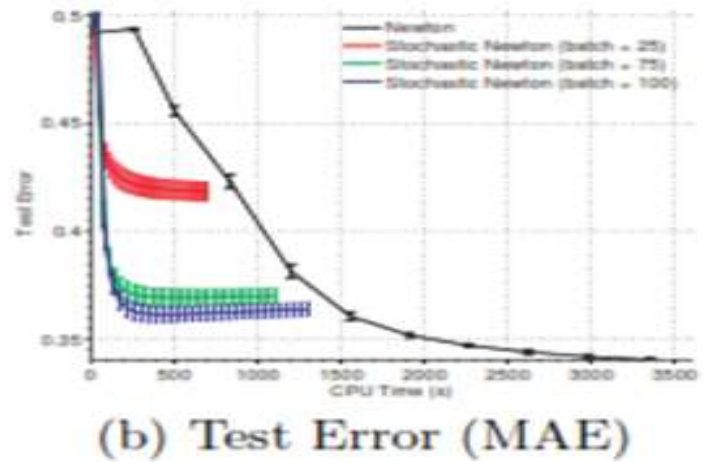

BehaviourofNewtonvs.StochasticNewtononathreefactormodel.

\section{CONCLUSION}

In this paper, we propose a novel sturdy multi-view Means clustering approach to solve the trouble of huge-scale multi-view clustering. Utilizing a commonplace cluster indicators, we can are trying to find consensus and do now not classify the patterns in some of the perspectives of visible functions. In addition, via enforcing a based sparse '12-1-norm within the goal function, our approach is robust to outliers within the facts input. Our new technique of gaining knowledge of the burden of every adaptive display. We additionally added an optimization algorithm for iterative and efficiently solve non-smooth proposed through the convergence objective proved. We examine the performance of our approach on six multi-view clustering statistics units.

\section{REFERENCE}

1. [Bay et al., 2008] Herbert Bay, Andreas Ess, laars TinneTuyte-, and Luc J. Van Gool. Speeded-up tures a strong constraint at the thing (browsing). Computer Vision and Image Understanding, 110 (3): 346-359, 2008.

2. [Biswas and Jacobs, 2012] ArijitBiswas and David Jacobs. Active Image Clustering: Finding Constraints of human mans Complementary to the set of rules. CVPR, pages 2152-2159, 2012.

3. Three. [Bosch et al., 2007] Anna Bosch, Andrew Zisserman, and Xavier Mun oz.. Representing shape with spatial pyramid kernel. In CIVR, pages 401-408, 2007.

4. [Cai et al., 2011] Xiao Cai, FeipingNie, Heng Huang, and FarhadKamangar. Tion agency picture feature heterogeneous multi-modal integration through spectral clustering. In IEEE Confer- ence on Computer Vision and Pattern Recognition (CVPR 2011), pages 1977-1984, 2011.

5. Five. [Chang and Lin, 2011] Chih-Chung Chang and Chih-Jen Lin. Libsvm: A library for assist vector machines. ACM tist, 2 (three): 27, 2011.

6. 6. [Dalal and Triggs 2005] NavneetDalal and Bill Triggs. His tograms orientated gradients for human detection. In CVPR (1), pages 886-893, 2005.

7. 7. [Ding et al., 2005] Chris H. T. Ding Xiaofeng He, and Horst D. Simon. Lagrangian relaxation isn't poor ok means and spectral clustering. In the ECML, pages 530 538, 2005. 
8. Eight. [Duarte and Hu, 2004] Marco F. Duarte and Yu Hen Hu. Ve- hicle category in dispensed sensor networks. J. Par- allelDistrib. Comput, sixty four (7) :. 826-838, 2004.

9. 9. [Dueck and Frey 2007] Delbert Dueck and Brendan J. Frey. Affinity propagation of non-metric for unattended photo categorization. In ICCV, pages 1-8, 2007.

10. 10. [Frank and Asuncion 2010] A. Frank and A. Asuncion. 2010 UCI system learning repository. 